\title{
Excitonic Behavior of Rhodamine Dimers: A Single-Molecule Study
}

\author{
Jordi Hernando, ${ }^{*},{ }^{\dagger}$ Martijn van der Schaaf, ${ }^{\dagger}$ Erik M. H. P. van Dijk, ${ }^{\dagger}$ Markus Sauer, ${ }^{\dagger}$ \\ María F. García-Parajó, ${ }^{\dagger}$ and Niek F. van Hulst ${ }^{\dagger}$
}

\author{
Applied Optics Group, Department of Applied Physics and MESA ${ }^{+}$Research Institute, University of Twente, \\ P.O. Box 217, 7500 AE Enschede, The Netherlands, and Physikalisch-Chemisches Institut, Universität \\ Heidelberg, Im Neuenheimer Feld 253, 69120 Heidelberg, Germany
}

Received: August 19, 2002; In Final Form: October 22, 2002

\begin{abstract}
The optical behavior of a dimer of tetramethylrhodamine-5-isothiocyanate has been investigated by means of single-molecule measurements. Bulk absorption and fluorescence spectra show the existence of two populations of the dimer molecule that exhibit distinct excitonic interactions (strong and weak coupling). Fluorescence confocal scanning microscopy has been employed to analyze the behavior of the weakly coupled dimers at the single-molecule level. Stepwise photodamage and collective on/off behavior have been observed in realtime fluorescence trajectories of the dimer. By polarization-sensitive detection, we distinguished between two conformationally different subpopulations within the weakly interacting dimers. Correlation between the fluorescence intensity and fluorescence lifetime of the dimer recorded in time has revealed the competition between photobleaching and trap formation as photodamaging processes of the chromophores in the dimer. The results obtained demonstrate the capability of single-molecule techniques to provide detailed insight into the exciton dynamics in multichromophoric systems, which is a key point in understanding the behavior of relevant natural aggregates and, eventually, in allowing for a rational design of molecular photonic devices.
\end{abstract}

\section{Introduction}

The study of optical phenomena in multichromophoric molecular aggregates is an issue of current interest because of the intriguing properties displayed by such systems and their potential applications in molecular electronics and photonics. For instance, biologically and chemically relevant systems such as light-harvesting antennas ${ }^{1}$ and $J$ aggregates ${ }^{2}$ show optical behavior that is governed by excitonic interactions between multiple chromophores. In recent years, single-molecule spectroscopy (SMS) has proven to be a powerful technique with which to study exciton dynamics in multichromophoric systems. ${ }^{3-25}$ Thus, stepwise photobleaching of the chromophores in the aggregate and the existence of collective on/off states, features that cannot be investigated by means of conventional experiments probing the averaged behavior of large ensembles of molecules, have been demonstrated at the single-molecule level.

Until now, most of the SMS studies on multichromophoric aggregates have focused on complex systems containing a large number of chromophore units such as the light-harvesting complex LH2 from Rhodopseudomonas acidophila ${ }^{1,3-6}$ and Pisum savitum, ${ }^{7}$ the photosynthetic pigment B-phycoerytrin from Porphyridium cruentum, ${ }^{8-9}$ the photosystem I complex from Synechococcus elongatus, ${ }^{10}$ and a multichromophoric conjugated polymer. ${ }^{1-15}$ Furthermore, other natural multichromophoric systems containing a smaller number of chromophore units such as the autofluorescent protein DsRed ${ }^{16-18}$ and the allophycocyanin trimer from Banana variabilis ${ }^{19}$ have been investigated at the single-molecule level. Although a wealth of information

* To whom correspondence should be addressed: E-mail: j.hernando@ tn.utwente.nl.

$\dagger$ University of Twente.

$\doteqdot$ Universität Heidelberg. has been obtained for the aforementioned aggregates, it has not been possible to rationalize their collective behavior fully because of the intrinsic complexity of those systems. Alternatively, some recent approaches have been devoted to the analysis of collective effects at the single-molecule level in simpler artificial multichromophoric systems, which are intended to mimic the behavior of natural aggregates. Energy transfer for a donor-acceptor pair has been investigated by using DNA as a rigid spacer and varying the interchromophore distance. ${ }^{20}$ Dendrimers have been employed to generate multichromophoric assemblies containing either one $\mathrm{e}^{9,21-23}$ or two ${ }^{24}$ different types of chromophore units, and their optical behavior has been studied at the single-molecule level. Very recently, an initial analysis of perylene dimers has been reported. ${ }^{25}$ Unfortunately, the spatial distribution of the chromophores in most aggregates is usually ill-defined, preventing a full understanding of the excitonic behavior.

In this work, we have combined ensemble and singlemolecule optical detection to investigate thoroughly the excitonic behavior of a well-defined multichromophoric system: a synthesized dimer of the tetramethylrhodamine-5-isothiocyanate dye (5-TRITC). While ensemble experiments show the existence of two populations of the dimer exhibiting different excitonic interactions, the inherent individuality of single-molecule analysis uncovers the presence of different subpopulations within weakly interacting dimers. The accurate control of the spatial arrangement of multichromophoric systems combined with single-molecule detection techniques allows for an ultimate comprehension of the excitonic behavior and, eventually, will open the possibility for the rational design of truly molecular photonic devices. 


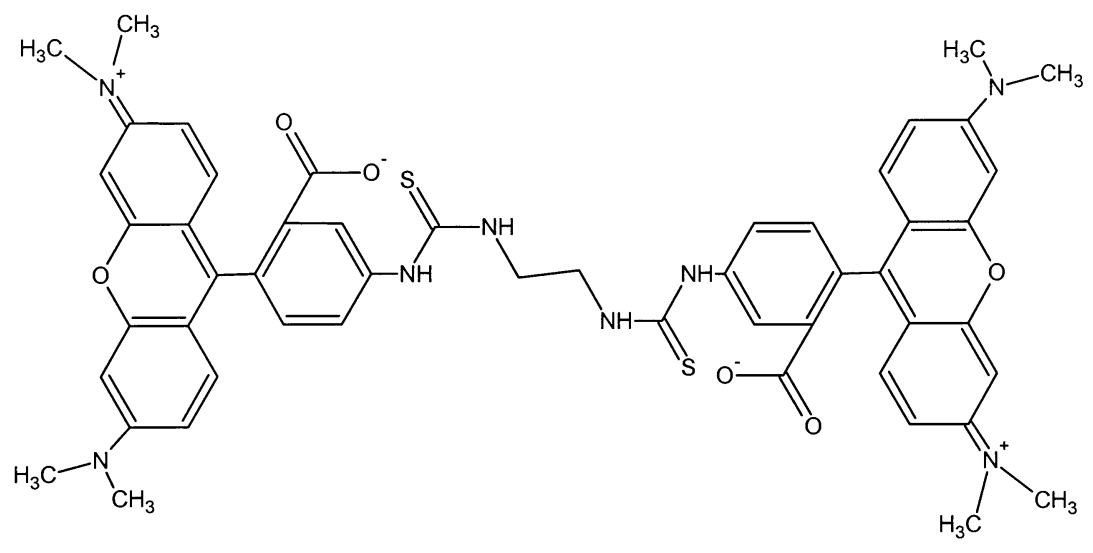

$\mathbf{a}$

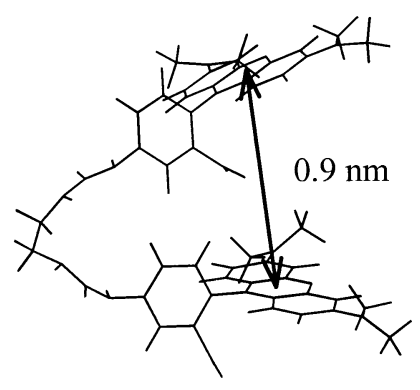

b

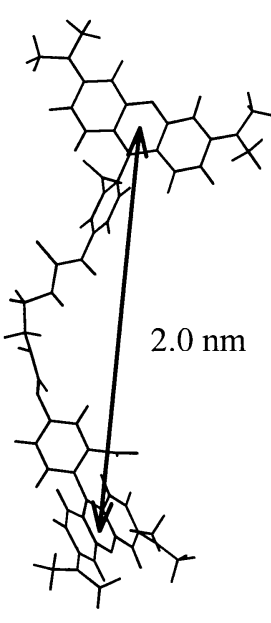

c

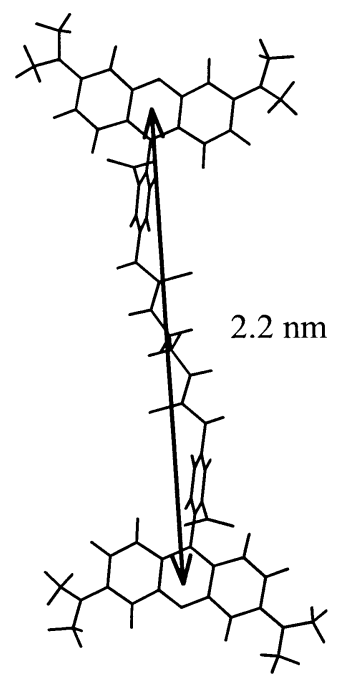

d

Figure 1. (a) Structure of the $\mathrm{TRITC}_{2}$ dimer. Optimal geometric configurations calculated for the dimer at the MNDO level: (b) folded, (c) gauche, and (d) extended. The calculated center-to-center dipole-dipole distances for the two rhodamine units in the different geometries are given.

\section{Experimental Section}

The TRITC dye was purchased from Molecular Probes and employed without further purification to synthesize the tetramethylrhodamine dimer (TRITC ${ }_{2}$, Figure 1a). 1,2-Diaminoethane was used as linker between the two TRITC units. Since this linker provides some flexibility in terms of the arrangement between the two chromophores composing the dimer, MNDO calculations were carried out by means of the Gaussian 98 package of programs ${ }^{26}$ to elucidate the different possible optimal geometries of the system in vacuum. Figure $1 b-d$ shows the three most stable minimal configurations considering the rotation of the TRITC units around the ethylene linker. Two limiting arrangements are possible: a "folded" geometry (Figure 1b) and an "extended" geometry (Figure 1d). An intermediate situation with the two chromophores in the ethylene linker arranged in a "gauche" configuration was also obtained (Figure 1c).

Bulk steady-state measurements were performed on solutions of TRITC and TRITC $_{2}\left(\sim 10^{-7} \mathrm{M}\right)$ in water (Aldrich, HPLC grade) containing $10 \mathrm{mg} / \mathrm{mL}$ (1 wt \%) poly(vinyl alcohol) (PVA, Aldrich, 99\%) and methanol (Merck, 99.9\%) and a 99:1 mixture of toluene (Fisher, 99.9\%)/methanol (the dyes used are not soluble in pure toluene). Absorption spectra were measured on a Shimadzu UV-2101PC spectrophotometer. Fluorescence spectra were recorded on an Edinburgh FS900 fluorospectrophotometer.

Samples for the single-molecule measurements were prepared by spin coating solutions of TRITC and TRITC $_{2}\left(\sim 10^{-10} \mathrm{M}\right)$ in water containing $10 \mathrm{mg} / \mathrm{mL}$ PVA on a cover glass at 3500 rpm for $90 \mathrm{~s}$. Thin polymer films 20 -nm thick as measured by AFM were obtained. The cover glasses were cleaned by means of an $\mathrm{O}_{2}$-etching plasma treatment for $5 \mathrm{~min}$ before sample preparation. Only freshly prepared samples were used in the single-molecule measurements.

Single-molecule experiments were carried out using a confocal scanning fluorescence microscope (CFM, Zeiss Axiovert inverted microscope) with an oil-immersion objective lens (Olympus NA 1.4, 100 $\times$ ). ${ }^{27}$ Circularly polarized light coming from an $\mathrm{Ar}^{+} / \mathrm{Kr}^{+}$ion laser (Spectra Physics, BeamLok 2060) and from a frequency-doubled optical parametric amplifier (Spectra Physics, OPAL 1100-1300 nm, $500 \mathrm{~mW}$ ) pumped by a Ti:Sa laser (Spectra Physics, Tsunami model 3960, $775 \mathrm{~nm}$, $2.5 \mathrm{~W})$ was used to excite the dye molecules in a continuous $(\lambda$ $=514 \mathrm{~nm})$ and pulsed way $(\lambda=550 \mathrm{~nm}, 130 \mathrm{fs}$ pulses $)$. Bandpass excitation blocking filters were inserted at the entrance of the microscope $(\lambda=514 \mathrm{~nm}$, Omega $514.5 / 10 ; \lambda=550 \mathrm{~nm}$, Omega 550DF30). The fluorescence light emitted by the dye 

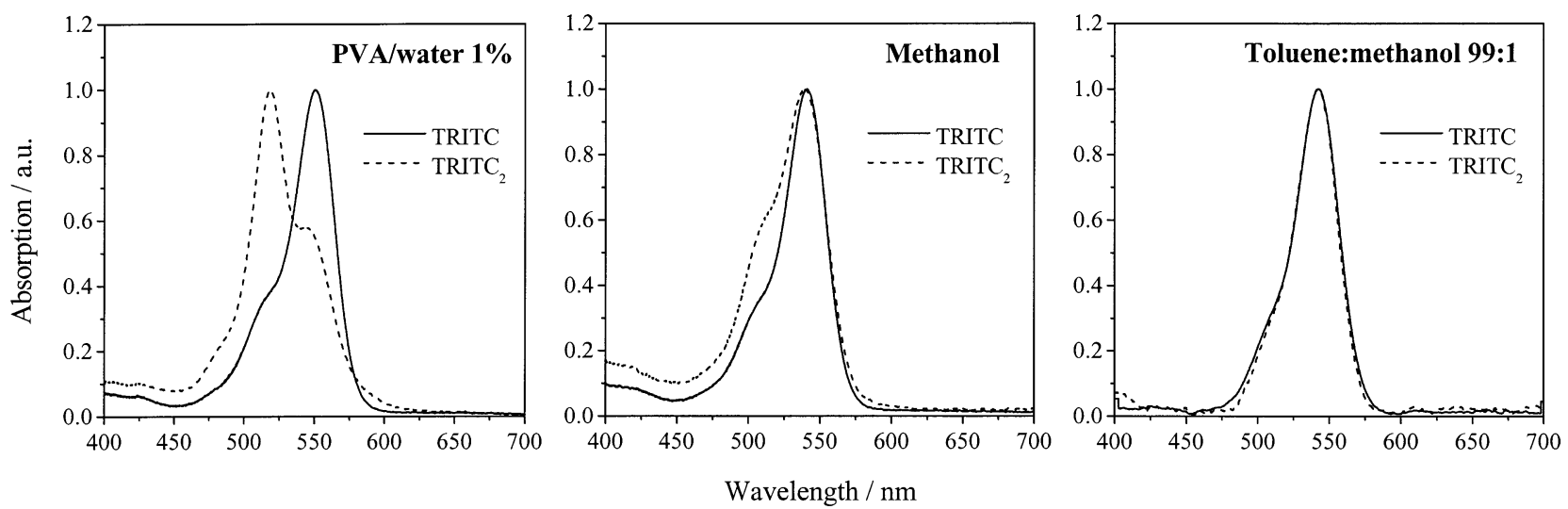

Figure 2. Absorption spectra of TRITC and TRITC $_{2}$ in 1 wt \% PVA/water, methanol, and 99:1 toluene/methanol.

was collected through the same objective lens, separated from the excitation light by the use of a dichroic mirror $(\lambda=514$ nm, Omega 540DRLP; $\lambda=550 \mathrm{~nm}$, Omega 565DRLP) and a rejection band or a long-pass filter $(\lambda=514 \mathrm{~nm}, 514.5$ Raman; $\lambda=550 \mathrm{~nm}$, Omega 570ALP0110), split into two orthogonal polarization components ( $\mathrm{s}$ and $\mathrm{p}$ components) by a polarizing beam-splitter cube, and directed to the small detection area (180$\mu \mathrm{m}$ diameter) of two avalanche photodetectors (SPCM-AQ-14, EG\&G Electro Optics). The whole setup was computer controlled by custom-made LabView software. For the pulsed excitation experiments, the signals from the APDs together with the trigger signal were collected using an SPC 500 timecorrelated single-photon counting card (Becker \& Hickl) to measure the arrival times of the photons to the detectors for sampling intervals of $60 \mathrm{~ms}$. Histograms of the photon arrival times were fitted to an exponential decay by means of a leastsquares analysis to determine the fluorescence lifetimes.

Fluorescence images of individual monomers and dimers were acquired by scanning the samples over areas of $5 \times 5 \mu \mathrm{m}^{2}$ or $10 \times 10 \mu \mathrm{m}^{2}$ at a pixel frequency of $1 \mathrm{kHz}$ and an excitation power of $1 \mathrm{~kW} / \mathrm{cm}^{2}$. The temporal dependence of the fluorescence intensity (fluorescence time trajectories), fluorescence polarization, and fluorescence lifetime for single monomers and dimers was measured by parking the laser beam on a particular fluorescence spot and recording the real-time fluorescence until irreversible photobleaching was observed (generally in less than $60 \mathrm{~s}$ ). An excitation power of $4.5 \mathrm{~kW} / \mathrm{cm}^{2}$ (well below the saturation limit) and a dwell time of $100 \mu$ s were employed to record the fluorescence trajectories.

\section{Results and Discussion}

Bulk Absorption and Fluorescence Spectra. Figure 2 shows the absorption spectra of both TRITC and TRITC $_{2}\left(\sim 10^{-7} \mathrm{M}\right)$ in 1 wt \% PVA/water, methanol, and a 99:1 mixture of toluene/ methanol. Over the range of $5 \times 10^{-6}$ to $5 \times 10^{-8} \mathrm{M}$, the recorded spectra were essentially independent of the concentration. While the shape of the spectra for the monomer is independent of the solvent, different features are observed for the three dimer solutions. For the polar PVA/water solution, the absorption spectrum of the dimer has a blue-shifted peak partially overlapping the absorption band of the monomer. The contribution of the blue-shifted peak is much less pronounced for the methanol solution. For the nonpolar toluene solution, the absorption spectrum of the dimer is the same as that for the monomer.

Figure 3 shows the emission fluorescence spectra of both TRITC and TRITC $_{2}$ in the PVA/water and methanol solutions, respectively. In contrast to the differences observed in the absorption spectra, monomer and dimer solutions display the same emission spectra. Moreover, the shape of the dimer spectra is independent of whether the excitation wavelength corresponds to the monomer absorption maximum (550 and $541 \mathrm{~nm}$ for the $\mathrm{PVA} /$ water and methanol solutions, respectively) or to that of the blue-shifted peak in the dimer spectra (519 and $510 \mathrm{~nm}$ for the PVA/water and methanol solutions, respectively). For the dimer sample in PVA/water, the emission intensity ratio for these two wavelengths $\left(I_{519} / I_{550}\right)$ is 0.43 whereas the absorption ratio $\left(A_{519} / A_{550}\right)$ is 1.5 . However, the $A_{519} / A_{550}$ ratio for the monomer in PVA/water is 0.47 . Similar behavior is observed for the samples in methanol. This suggests that there are two different absorbing species of the dimer: one of them having the same emission spectrum as the single TRITC and the second one being essentially nonfluorescent. This hypothesis was proven by measuring the excitation fluorescence spectra. The results are also shown in Figure 3 and compared with the absorption spectra. Clearly, the emitting species has the same absorption as the monomer, and thus the nonfluorescent species is responsible for the blue-shifted absorption band.

Since the absorption spectra of TRITC $_{2}$ in water and methanol are concentration-independent, self-aggregation of the dye leading to blue-shifted nonfluorescent species as reported for highly concentrated rhodamine solutions ${ }^{28}$ and gels ${ }^{29}$ can be ruled out. Therefore, the observed behavior arises from the existence of different conformations of the $\mathrm{TRITC}_{2}$ molecule with different optical properties. In fact, we theoretically predicted the existence of multiple conformations due to the flexibility of the tether linking the two rhodamine units (see Experimental Section). As multiple geometrical conformations have different interchromophoric distances, different types of interactions might be established between the chromophore units, resulting in distinct optical properties.

Two limiting cases can be distinguished for the excitonic interaction in a bichromophoric system with identical chromophores. ${ }^{30}$ First, for short interchromophoric distances, the strong Coulombic interaction between the chromophore units results in a Davydov shift/splitting of the absorption spectrum and an enhancement ( $\mathrm{J}$ dimer) or loss $(\mathrm{H}$ dimer) of the emission. ${ }^{31}$ In this case, the excitation energy is delocalized within the entire bichromophoric system, which behaves as a new single quantum system with a new ground state and two new excited excitonic states. Second, for larger distances, the weak interaction between the chromophore units does not alter the absorption spectrum, but it allows for incoherent energy transfer between the units. This energy-transfer process is usually described by means of Förster theory, ${ }^{32}$ which assumes a transition dipole-dipole interaction scheme. However, other 

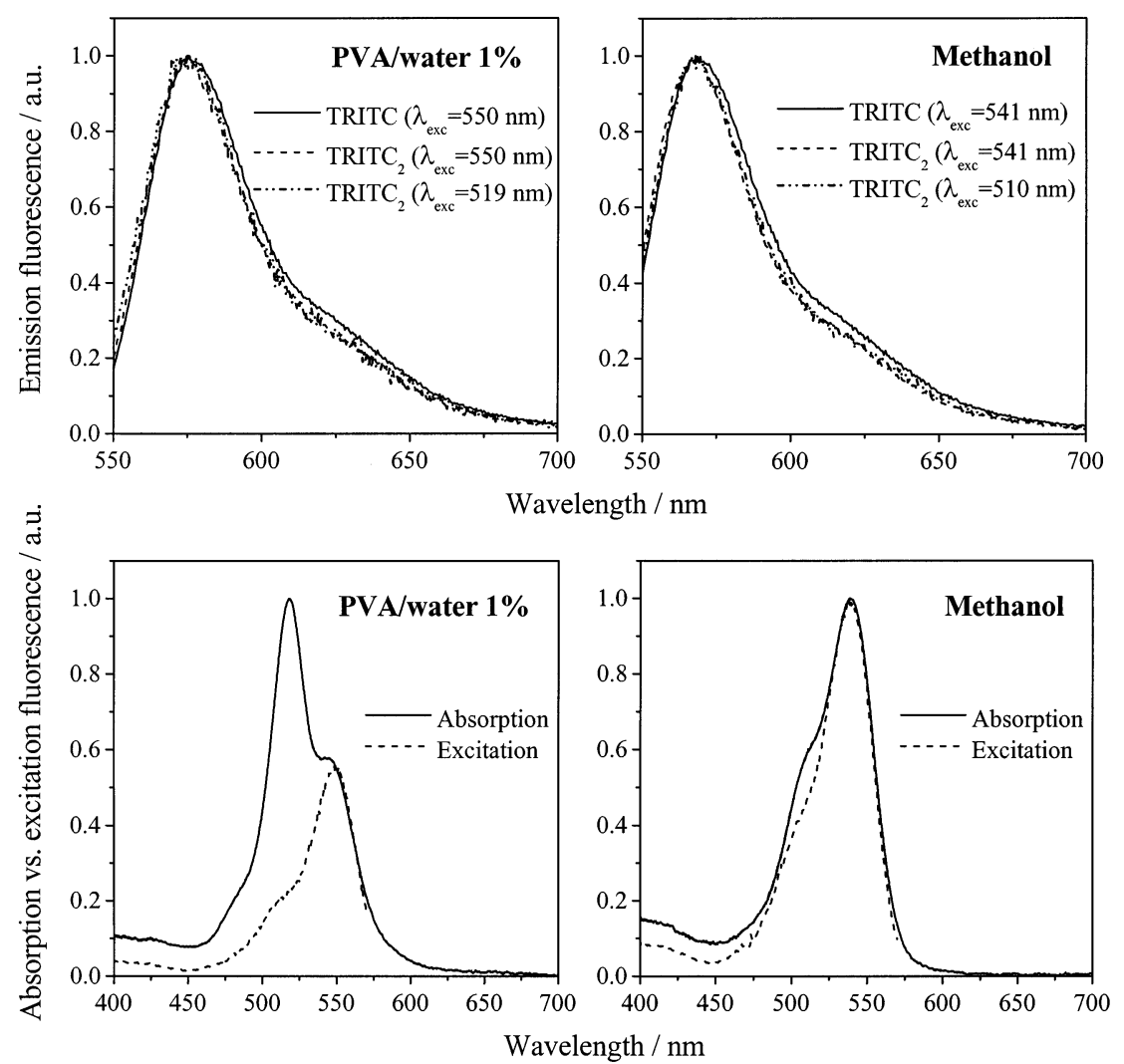

Figure 3. Emission and excitation fluorescence spectra of TRITC and $\mathrm{TRITC}_{2}$ in PVA/water and methanol. The excitation fluorescence spectra are compared with the absorption spectra.

coupling mechanisms can account for the energy-transfer mechanism between weakly coupled chromophore units, such as non-Coulombic Dexter exchange coupling ${ }^{33}$ or a Coulombic interaction arising from the spatial proximity between the donor and acceptor units even when the transition moment of one of them is zero. The latter has been recently proposed to explain the energy transfer between the $S_{1}$ state of carotenoids and bacteriochlorophylls in light-harvesting complexes. ${ }^{34}$

Considering the optimal geometrical conformations as calculated for the TRITC $_{2}$ dimer (Figure 1), a significant shift between the dimer and the monomer absorption peaks is expected only for the geometry shown in Figure $1 b .{ }^{30}$ Specifically, a 21-nm blue shift is calculated, which compares reasonably with the $31-\mathrm{nm}$ shift determined between the blueshifted dimer and the monomer peaks in the absorption spectra in PVA/water. Moreover, in the case of strong coupling effects, molecule $1 \mathrm{~b}$ is expected to be nonfluorescent because of the nearly parallel arrangement of the transition dipoles of both chromophore units ( $\mathrm{H}$ dimer). Using this information, the first assignment between the two TRITC $_{2}$ species detected in the experiments and the calculated conformations is made. The molecules of TRITC 2 adopting folded geometries close to that depicted in Figure 1b are responsible for the appearance of a blue-shifted band in the absorption spectrum that yields no fluorescence. However, the molecules in more extended conformations such as the ones shown in Figure 1c and d display the same absorption and emission spectra as the monomer because only weak interchromophoric interactions take place.

The number of strongly and weakly coupled TRITC $_{2}$ molecules depends on the solvent, as evidenced by the absorption spectra shown in Figure 2. The more polar the solvent, the larger the tendency to form folded $\mathrm{H}$ dimers. The lack of eclipsed conformations in the tether should make the extended geometries more stable for solutions with nonpolar solvents, whereas strong solvent-solvent interactions for polar solvents should promote a minimization of the solute area to be solvated, thus favoring the formation of folded conformations (solvophobic effect). This situation has already been described for squaraine ${ }^{35}$ and merocyanine dimers. ${ }^{36}$

Although the first insight into the excitonic behavior of the rhodamine dimer has been attained by means of steady-state bulk measurements, several aspects remain unclear. This is mainly due to the complexity of the system since different species are possible for the dimer depending on the arrangement of the two dye units. Single-molecule detection appears then to be an ideal technique to investigate the behavior of the rhodamine dimer further, as it has been shown to uncover the spatial and temporal inhomogenity of populations..$^{37,38}$ Thus, dimer molecules with different relative orientations of both chromophores can be distinguished at the single-molecule level, their optical properties then being studied separately. Moreover, the analysis of the temporal evolution of these properties by means of SMS should allow for a better understanding of the excitonic interaction taking place between the two dye units in the aggregate.

Single-Molecule Measurements. Figure $4 \mathrm{a}$ and $\mathrm{b}$ shows fluorescence images of individual monomers and dimers of rhodamine. Two examples of fluorescence intensity trajectories for single TRITC and TRITC $_{2}$ molecules are given in Figure $4 \mathrm{c}$ and $\mathrm{d}$. Typical single-molecule features such as the transition from emissive (bright) to nonemissive (dark) states (the so-called blinking) and the final cessation of emission due to irreversible photobleaching are observed. Longer survival times and a larger number of emitted photons were found for the dimer molecules with both continuous and pulsed irradiation. Using CW excitation light of $514 \mathrm{~nm}\left(4.5 \mathrm{~kW} / \mathrm{cm}^{2}\right.$ at the sample), TRITC and TRITC $_{2}$ molecules have average survival times of 11 and $17 \mathrm{~s}$, respectively. Fitting monoexponential functions to the histo- 

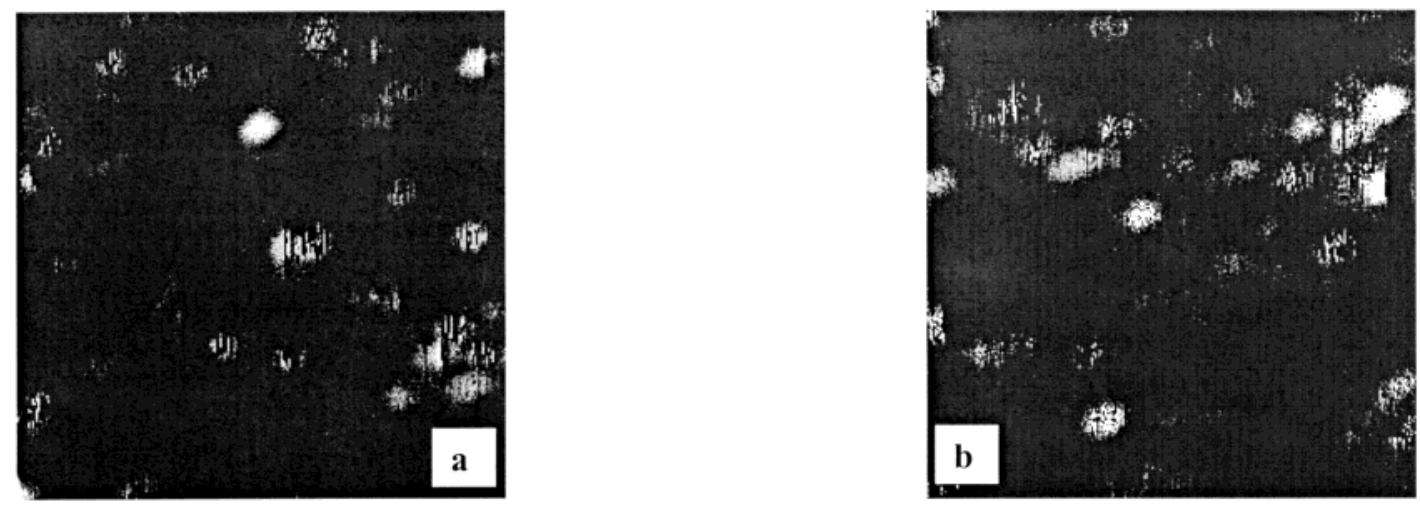

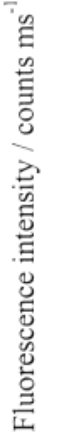
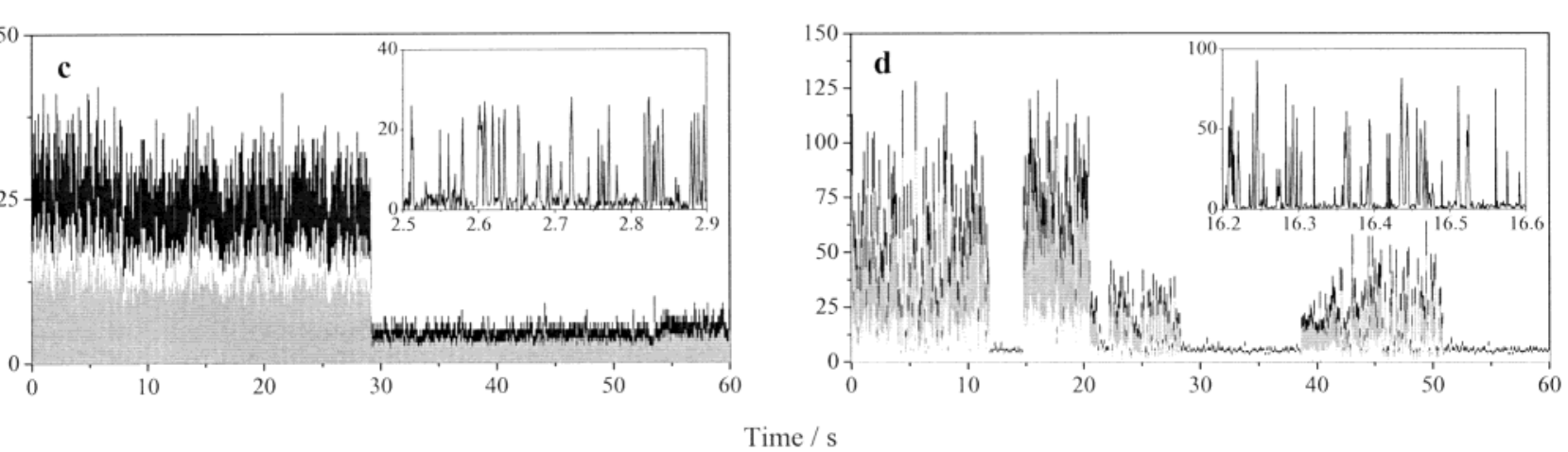

Figure 4. Fluorescence images $\left(5 \times 5 \mu \mathrm{m}, \lambda_{\mathrm{exc}}=514 \mathrm{~nm}\right.$, power $=1 \mathrm{~kW} / \mathrm{cm}^{2}, 256 \times 256$ pixels, $1 \mathrm{~ms} /$ pixel $)$ and time trajectories $\left(\lambda_{\text {exc }}=550\right.$ $\mathrm{nm}$, power $\left.=4.5 \mathrm{~kW} / \mathrm{cm}^{2}\right)$ of $(\mathrm{a}, \mathrm{c})$ TRITC and $(\mathrm{b}, \mathrm{d})$ TRITC $_{2}$ single molecules embedded in a PVA matrix. In the fluorescence time trajectories, the black lines stand for the total fluorescence signal, and the gray lines stand for the two orthogonal polarization components of the emission. The insets zoom in on a small time window in the trajectories.

grams of the number of photons emitted at this condition leads to characteristic numbers of detected photons before photobleaching equal to $3.4 \times 10^{5}$ and $6.5 \times 10^{5}$ photons for TRITC and TRITC $_{2}$, respectively. These results are consistent with the dimer molecules having two chromophore units.

A. Fluorescence Intensity Trajectories: TRITC versus TRITC $_{2}$. One of the typical properties of single dye emitters is the one-step photobleaching behavior. Only two levels of intensity corresponding to emissive and nonemissive states are usually observed. Around $80 \%$ of the TRITC monomers analyzed for both continuous and pulsed excitation exhibit this behavior, as clearly seen in the time trajectory depicted in Figure $4 \mathrm{c}$. The combined normalized intensity histogram ${ }^{12}$ for 121 TRITC molecules studied by continuous excitation indeed shows two peaks (see Figure 5a). The first peak is the background level; the second one corresponds to the highest emissive intensity level. The large spread of the second peak in the histogram is partially due to the stressed blinking behavior of rhodamine timetraces, as clearly seen in Figure 4c. Also, the existence of some molecules with multiple intensity levels accounts for the broadening of the histogram. For a few of them, the fluctuations in the emission intensity correlate with changes in the emission polarization, indicating rotational jumps. Around $15 \%$ of the fluorescence trajectories analyzed show intensity fluctuations that do not correlate with changes in the emission polarization. They should be attributed to changes in the photophysical properties of the emitters during the detection time. This situation has already been reported for singlemolecule experiments on single rhodamine dyes and has been assigned to the existence of spectral jumps arising from either variations in the nanoenvironment surrounding the molecules or photochemical processes. ${ }^{39-41}$
The combined intensity histogram of 172 individual TRITC $_{2}$ molecules studied by continuous excitation at $\lambda_{\mathrm{exc}}=514 \mathrm{~nm}$ is depicted in Figure 5b. In this case, three different peaks are observed: the first one is the background level, and the others correspond to two different emissive levels. This situation accounts for the fact that most of the TRITC $_{2}$ fluorescence trajectories show two distinct fluorescence intensity levels $(65 \%)$, as shown in Figure $4 d$. This arises as a consequence of the sequential photodamage of the two chromophores units in the dimer. $5,7,9-19,21-25$ Initially, both chromophores can absorb and emit; after repetitive excitation, one of them is photobleached so that only the remaining dye unit is emitting and thus a lower intensity level is detected. For about half of the trajectories showing two different intensity levels (34\% of the total), reversible jumps were observed between them. This means that reversible photodamage of one of the two chromophores can take place before irreversible photobleaching.

The ratio of the two emissive peaks $\left(I_{2} / I_{1}\right)$ in the TRITC $_{2}$ intensity histogram is 0.42 . The slight deviation with respect to the 0.5 ratio expected for equidistant intensity levels can be explained in two different ways: (a) orientational effects arising from the two dye units not being arranged in-plane and (b) the photodamaged chromophore partially quenches the fluorescence of the remaining emitting one. These two points will be discussed further in sections $b$ and e, respectively.

The aforementioned reasons can also explain why $35 \%$ of the TRITC $_{2}$ fluorescence trajectories show only one emissive level. Premature photobleaching of one of the chromophore units before recording the trajectories is unlikely to account for such a large number of molecules displaying only one intensity level. On average, we imaged a molecule for $\sim 200 \mathrm{~ms}$ whereas the mean duration of the highest intensity level in the trajectories 


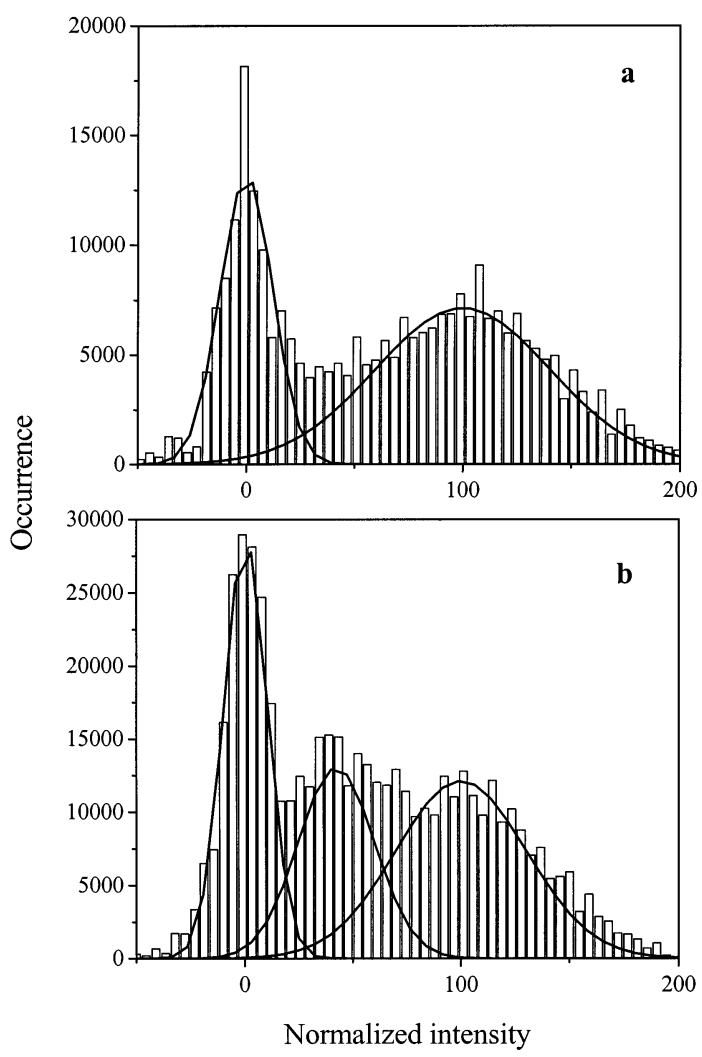

Figure 5. Combined normalized-intensity histogram of (a) 121 TRITC and (b) 172 TRITC $_{2}$ single molecules $\left(\lambda_{\text {exc }}=514 \mathrm{~nm}\right.$, power $=4.5$ $\mathrm{kW} / \mathrm{cm}^{2}$ ). Each trajectory was normalized to the mean intensity value of the highest emissive level. Gaussian functions have been fitted to the peaks in the histograms.

is about $800 \mathrm{~ms}$. In fact, only $6 \%$ of the trajectories with two intensity levels have survival times for the highest level that are lower than $200 \mathrm{~ms}$. Furthermore, we used 4 times less power to image the molecules $\left(1 \mathrm{~kW} / \mathrm{cm}^{2}\right)$ than to record time trajectories $\left(4.5 \mathrm{~kW} / \mathrm{cm}^{2}\right)$.

In the study of the allophycocyanin trimer, Ying and $\mathrm{Xie}^{19}$ found that multiple emissive levels in the intensity trajectories arose as a consequence of weakly coupled chromophores whereas the emission from strongly coupled dye pairs vanished in a one-step process because of the creation of efficient photoinduced exciton traps. Therefore, the fact that most of the TRITC $_{2}$ intensity trajectories show two different emissive levels suggests that weak excitonic interactions take place between the two chromophore units for the fluorescent dimers. This is consistent with the results from the steady-state bulk measurements showing that the strongly coupled TRITC $_{2}$ dimers are essentially nonfluorescent and thus that they cannot be studied by means of fluorescence microscopy. For this reason, it is very unlikely that the small number of TRITC $_{2}$ molecules showing a one-step photobleaching process correspond to strongly coupled dimers and also to weakly coupled dimers. In fact, onestep photobleaching behavior has also been reported for a small percentage of weakly coupled perylene dimers. ${ }^{25}$

B. Orientational Effects in the TRITC $_{2}$ Fluorescence Intensity Trajectories. As already mentioned, $65 \%$ of the TRITC $_{2}$ fluorescence trajectories show two emissive intensity levels that arise from the stepwise photobleaching of the two dye units. Figure 6 a shows a histogram of the intensity ratio $\left(I_{2} / I_{1}\right)$ between both levels for 111 TRITC $_{2}$ dimers studied at $\lambda_{\text {exc }}=514 \mathrm{~nm}$. For most of the molecules, the value of the intensity ratio is around $0.5\left(0.4<I_{2} / I_{1}<0.6\right.$ for $63 \%$ of the
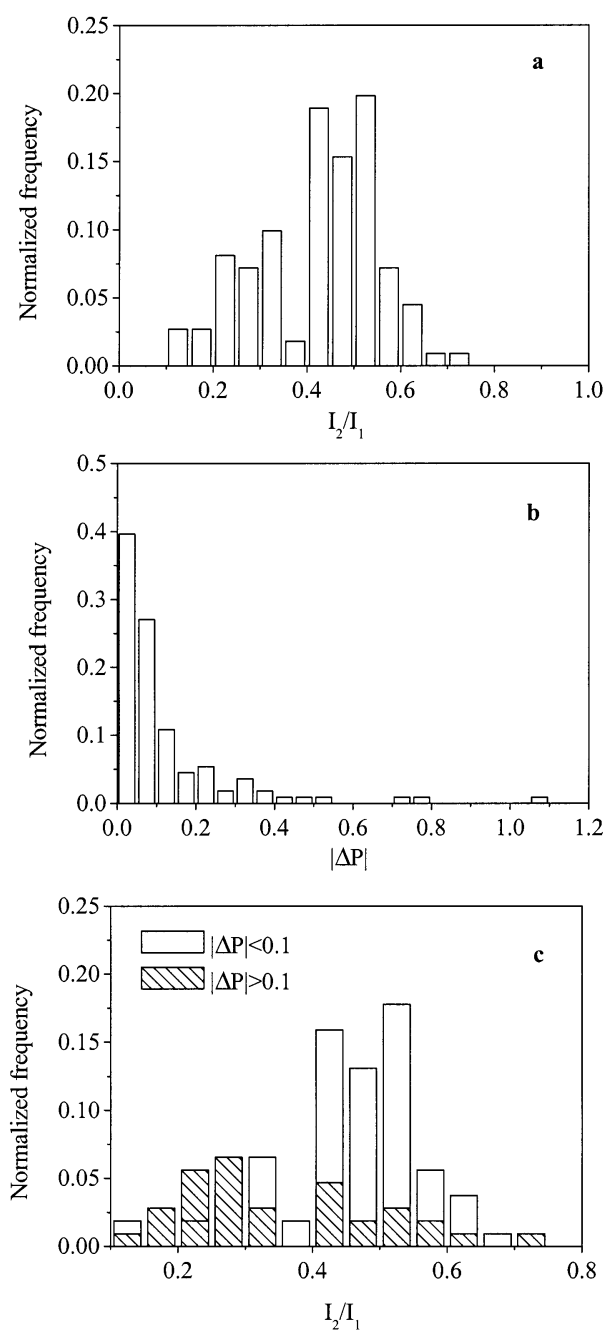

Figure 6. (a) Histogram of the intensity ratios between the two intensity levels $\left(I_{2} / I_{1}\right)$ for $111 \mathrm{TRITC}_{2}$ molecules $\left(\lambda_{\mathrm{exc}}=514 \mathrm{~nm}\right.$, power $=4.5$ $\mathrm{kW} / \mathrm{cm}^{2}$ ). (b) Histogram of the differences in linear polarization $(|\Delta P|)$ between the two intensity levels for the same set of molecules. (c) Histogram of $I_{2} / I_{1}$ for TRITC $_{2}$ molecules with $|\Delta P|<0.1$ and $|\Delta P|>$ 0.1 .

molecules), which agrees with the average value of 0.42 determined from the global intensity histogram in Figure 5a. However, a nonnegligible number of molecules display intensity ratio values clearly below $0.5\left(I_{2} / I_{1}<0.4\right.$ for $31 \%$ of the molecules). Similar values are found when using pulsed excitation at $550 \mathrm{~nm}$, although the percentage of molecules with low $I_{2} / I_{1}$ values was slightly larger $\left(I_{2} / I_{1}<0.4\right.$ for $38 \%$ of the 93 analyzed TRITC $_{2}$ molecules). The deviation from the ideal 0.5 value may be explained in terms of orientational effects arising from the two dye units in the aggregate not being arranged in-plane.

To analyze the effects of orientational factors on the intensity ratio, the change in the emission polarization between both intensity levels has been studied. The linear polarization $P$ of the emission was calculated for every intensity level according to $P=\left(I_{\mathrm{s}}-I_{\mathrm{p}}\right) /\left(I_{\mathrm{s}}+I_{\mathrm{p}}\right)$, and the difference between $P_{1}$ and $P_{2}$ values for the two intensity levels $I_{1}$ and $I_{2}$ was determined for each fluorescence trajectory. The histogram of the absolute values of this difference $\left(|\Delta P|=\left|P_{1}-P_{2}\right|\right)$ is plotted in Figure $6 \mathrm{~b}$ for $111 \mathrm{TRITC}_{2}$ molecules studied at $\lambda_{\mathrm{exc}}=514 \mathrm{~nm}$. Most of the molecules show little change in $P$ when jumping from one intensity level to the other $(|\Delta P|<0.1$ for around $70 \%$ of the molecules), implying that the two chromophores in TRITC $_{2}$ are mostly arranged parallel to each other. Nevertheless, a few 
molecules displayed large values of $|\Delta P|$, indicating a nonparallel disposition of the two dye units. The observation of these two different TRITC $_{2}$ subpopulations is consistent with the existence of different geometrical conformations of the dimer molecule, as described in the Experimental Section. In fact, we have calculated two different stable geometries in the case of the fluorescent, weakly coupled $\mathrm{TRITC}_{2}$ : an extended conformation with the two TRITC units arranged nearly parallel to each other and a gauche conformation with a nonparallel and an out-of-plane arrangement of the two chromophores. In the first case, $|\Delta P|$ values close to zero are expected whereas in the latter larger polarization differences must take place. Of course, the existence of only two possible geometries for the fluorescent $\mathrm{TRITC}_{2}$ species is an ideal situation, as they were calculated in vacuum without taking into account the interaction with the surrounding media. Therefore, a variety of additional configurations around the two limiting conformations might arise because of such an interaction. This easily explains the spread in the $|\Delta P|$ histogram.

To investigate the correlation between the $I_{2} / I_{1}$ and $|\Delta P|$ parameters, the intensity ratio histogram in Figure 6a has been split into two different components: intensity ratio histograms for TRITC $_{2}$ molecules with small $(|\Delta P|<0.1)$ and large $(|\Delta P|$ $>0.1)$ changes in polarization. The resulting plots are depicted in Figure 6c. Clearly, small fluctuations in $|\Delta P|$ correspond to intensity ratio values centered on 0.5 (mean value equal to 0.46 ). This is consistent with TRITC $_{2}$ molecules having the two chromophore units nearly parallel, provided that the bleaching of one of them does not quench the emission from the other. However, large variations in $|\Delta P|$ correlate with $I_{2} / I_{1}$ values below 0.5 (mean value equal to 0.36 ). If quenching is neglected, this effect is easily explained by the nonparallel dye units with an out-of-plane component for the nonextended TRITC $_{2}$ conformations, as theoretically predicted. For such a situation, both chromophores are not excited and detected with the same efficiency and therefore are not equally contributing to the total emission. The tendency to obtain $I_{2} / I_{1}$ values below 0.5 implies that there is a higher probability to bleach the chromophore unit that is in a more in-plane arrangement (i.e., the chromophore unit that is excited more efficiently). This is what should be expected a priori, as the photodamaging processes are directly connected to the formation of excited species.

C. Triplet Blinking and Long Dark States: TRITC versus TRITC $_{2}$. The TRITC fluorescence trajectory depicted in Figure $4 \mathrm{c}$ shows the typical on-off blinking behavior of single dye emitters. Short-lived dark states that exist during the emission of a single chromophore are usually considered to correspond to transitions to the triplet state (see the inset in Figure 4c). Assuming a three-level model, histograms of the duration of the off times for each molecule can be fitted to a monoexponential decay to determine the triplet lifetime, ${ }^{12,42}$ as shown in Figure 7a. Moreover, exponential fittings of the histograms of the number of photons emitted between adjacent off states allow for the determination of the intersystem-crossing yield $\left(Y_{\text {ISC }}\right),{ }^{12,42}$ as shown in Figure $7 \mathrm{~b}$. For a set of 70 TRITC molecules measured at $\lambda_{\mathrm{exc}}=514 \mathrm{~nm}$, we have built up the distributions of triplet lifetimes and intersystem-crossing yields, as plotted in Figure 7e and $\mathrm{f}$. The peak values of the triplet lifetime and $Y_{\text {ISC }}$ distributions for TRITC molecules in PVA are equal to $0.9 \mathrm{~ms}$ and $2.0 \times 10^{-3}$, respectively. In our analysis, a bin time of $500 \mu$ s was used, so triplet lifetimes shorter than this value could not be determined. This occurred for only $12 \%$ of the TRITC molecules. Moreover, some of the molecules showed
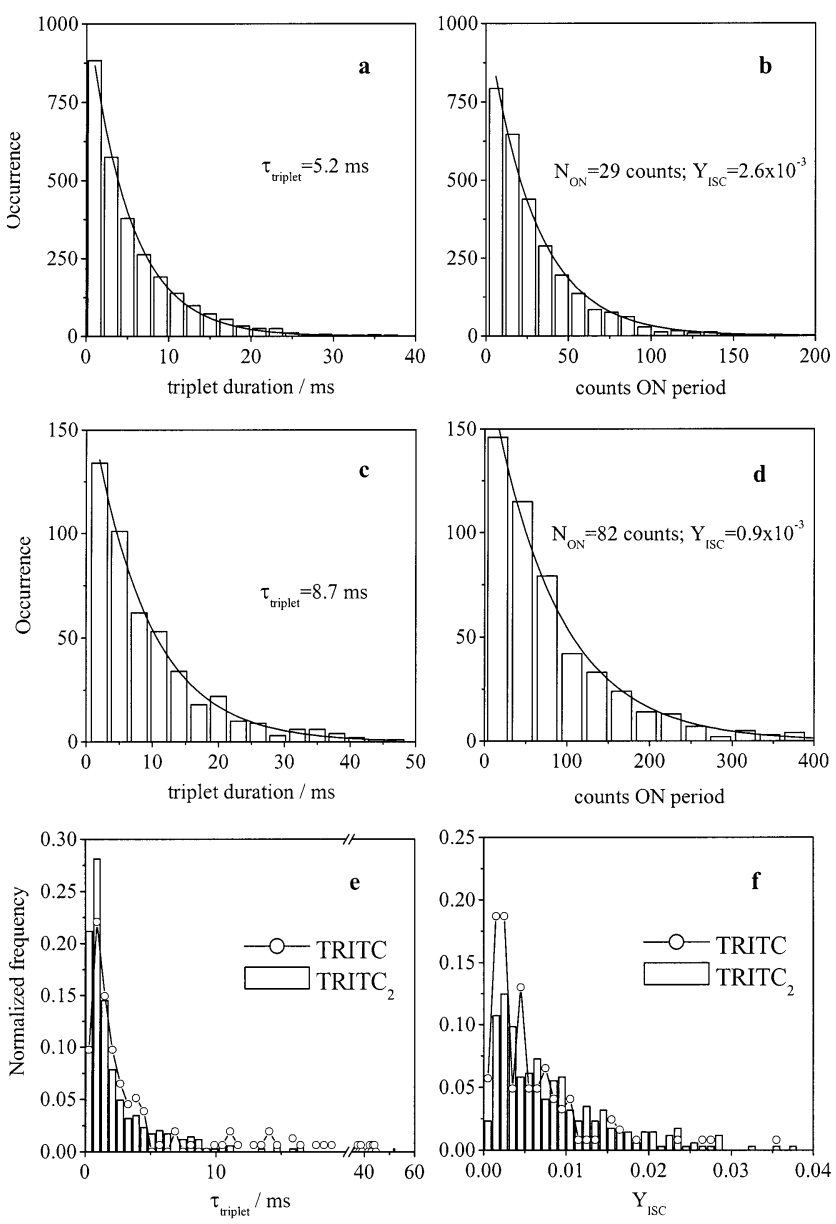

Figure 7. (a, b) Histograms of the duration of the triplet state and the number of counts between adjacent off periods for the TRITC trajectory depicted in Figure 4c; monoexponential functions were fitted to derive $\tau_{\text {triplet }}$ and $Y_{\text {ISC. }}$ (c, d) Same plots for the 15-21 s time interval in the TRITC $_{2}$ trajectory shown in Figure $4 \mathrm{~d}$. (e, f) Distributions of $\tau_{\text {triplet }}$ and $Y_{\text {ISC }}$ for 70 TRITC and 111 TRITC $_{2}$ molecules $\left(\lambda_{\text {exc }}=514 \mathrm{~nm}\right.$, power $\left.=4.5 \mathrm{~kW} / \mathrm{cm}^{2}\right)$.

changes in their triplet dynamics with time, which arise as consequence of fluctuations in the nanoenviroment of the molecules. ${ }^{42}$ In those cases, different values of the triplet lifetime and $Y_{\text {ISC }}$ were derived for the same molecule (54\%). Finally, triplet dynamics cannot account for long-lived dark states (ca. larger than $100 \mathrm{~ms}$ ), which are attributed to reversible photoinduced processes yielding nonemitting metastable conformations/derivatives of the molecule. Such dark states were observed in approximately $36 \%$ of the TRITC fluorescence trajectories (on average, 0.7 long dark states per time trajectory under our excitation conditions). By fitting an exponential decay to the distribution of long dark states, a characteristic time of $760 \mathrm{~ms}$ was found for TRITC.

The blinking behavior is also observed in the fluorescence trajectories of the TRITC 2 dimer. For instance, the inset in the TRITC $_{2}$ trajectory in Figure $4 d$ shows the existence of shortlived collective off periods for initial times when the two chromophore units are emitting. Interestingly, transitions from the highest intensity level to the background are observed. The histograms of the duration of the off states and of the number of photons for that molecule are well fitted to monoexponential decays, as shown in Figure $7 \mathrm{c}$ and d. Similar results were obtained for most of the measured fluorescence trajectories of TRITC $_{2}$, and only for a few of them $(11 \%)$ was the dynamics of the short off states not resolved due to the bin time employed 
$(500 \mu \mathrm{s})$. Figure $7 \mathrm{e}$ and $\mathrm{f}$ shows the comparison between the results obtained for the short-lived off periods of 111 TRITC $_{2}$ dimers and the triplet lifetime and $Y_{\text {ISC }}$ values determined for 70 TRITC monomers. For TRITC $_{2}$, the peak values of the distributions are $0.9 \mathrm{~ms}$ and $2.5 \times 10^{-3}$, respectively. These values do not significantly change if the two distinct intensity levels of the dimer molecules are analyzed separately since minor differences in the triplet dynamics are found when passing from the brightest (two emitting chromophores) to the dimmest (one emitting chromophore) level. This observation and the similarity between the overall histograms depicted in Figure 7 for both the monomer and the dimer support the assignment of the fast blinking in the TRITC $_{2}$ trajectories to the triplet dynamics of a single dye unit in the molecule. It has been proposed that once one of the chromophores of a multichromophoric aggregate visits the triplet it fully quenches the emission of the remaining dye units via a singlet-triplet mechanism, thus explaining the existence of short-lived collective off periods. ${ }^{9,23}$ Therefore, the observation of fast blinking is a clear sign that the two chromophore units in the dimer are close enough to interact. Our results demonstrate that the triplet dynamics of a rhodamine unit in the dimer is not significantly affected by the presence of an additional close interacting chromophore. This can be considered to be an additional indication that only weak coupling exists between the two dye units of the TRITC $_{2}$ molecules detected in our fluorescence microscopy experiments since for strongly coupled dimers behaving as a new quantum system differences in the triplet dynamics might be expected. ${ }^{30}$

We have also observed collective long-lived dark states for the TRITC $_{2}$ system, as seen in the trajectory depicted in Figure $4 \mathrm{~d}$, in agreement with other reports about multichromophoric aggregates. ${ }^{9,11-15,18,21}$ The few-seconds-duration dark state at $t$ $=12 \mathrm{~s}$ is unlikely to be due to a jump to the triplet state, but it should arise from another type of deactivation channel. The formation of a reversible photoinduced trap in one of the chromophores that quenches the fluorescence of the other one may explain this behavior. This trap might be a radical/cation or radical/anion pair generated in the molecule, as suggested by VandenBout et al., ${ }^{11}$ or just a metastable dark state of one of the chromophores, as is known for single dye emitters. Alternatively, another mechanism may account for long-lived dark states for the TRITC $_{2}$ system: a photoinduced change in the arrangement of the two chromophore units yielding a nonfluorescent conformation of the molecule. The combination of these different processes may explain why the number of long dark states increases for the dimer molecule with respect to the number for the monomer. For $\mathrm{TRITC}_{2}, 71 \%$ of the molecules show long dark states (on average, 2.1 dark states per molecule under our measurement conditions). By fitting an exponential decay to the distribution of long dark states, a characteristic time of $380 \mathrm{~ms}$ was found for TRITC 2 . This value is significantly lower than that determined for TRITC, which points toward the existence of different mechanisms of formation of the long dark states for both systems.

D. Fluorescence Lifetime: TRITC versus TRITC 2 . Fluorescence lifetime trajectories have been recorded for both the monomer and dimer. Figure 8 shows the distributions of fluorescence lifetimes measured at $\lambda_{\mathrm{exc}}=550 \mathrm{~nm}$ for 127 TRITC and 146 TRITC $_{2}$ molecules, respectively. The fluorescence lifetimes were derived from the monoexponential fitting of the overall photon arrival time histogram obtained for each molecule. Similar lifetime distributions with nearly equal average

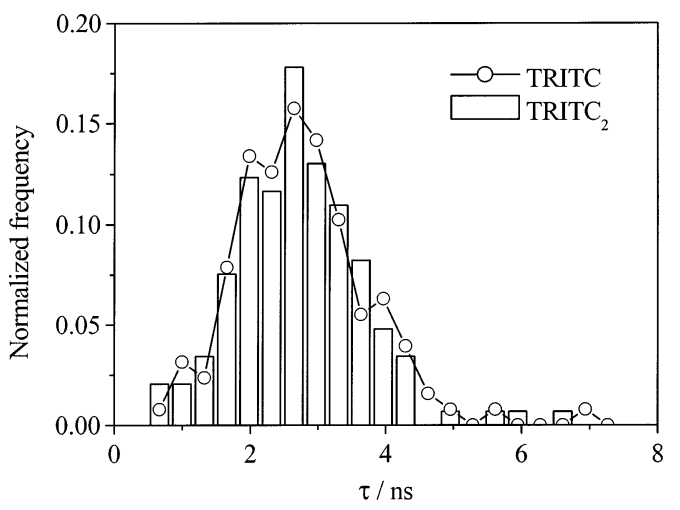

Figure 8. Distributions of fluorescence lifetimes for 127 TRITC and 146 TRITC $_{2}$ molecules $\left(\lambda_{\mathrm{exc}}=550 \mathrm{~nm}\right.$, power $\left.=4.5 \mathrm{~kW} / \mathrm{cm}^{2}\right)$.

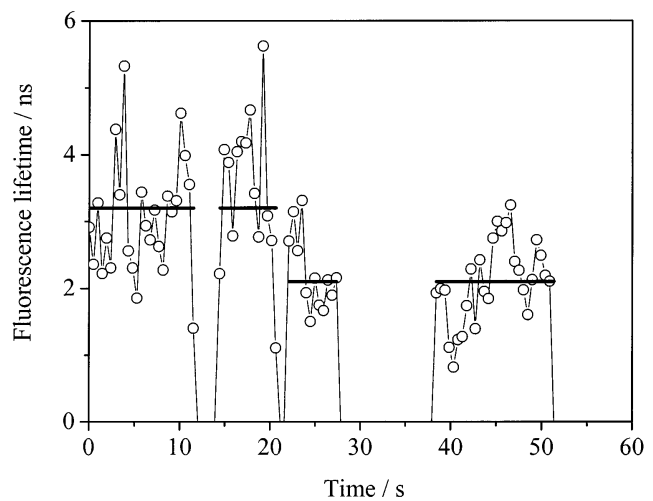

Figure 9. Fluorescence lifetime trajectory corresponding to the $\mathrm{TRITC}_{2}$ fluorescence intensity trajectory depicted in Figure 4d. A sampling interval of $480 \mathrm{~ms}$ has been used to fit the fluorescence lifetimes to photon time arrival histograms. The solid lines stand for the mean values of $\tau$ corresponding to both the higher and dimmer intensity levels (3.2 and $2.1 \mathrm{~ns}$, respectively).

values were determined for both types of samples: $\tau=2.6 \pm$ 0.8 and $2.7 \pm 0.8 \mathrm{~ns}$ for TRITC and $\mathrm{TRITC}_{2}$, respectively. Ensemble measurements in $1 \mathrm{wt} \% \mathrm{PVA} /$ water yielded similar results: $\tau=2.6$ and $2.4 \mathrm{~ns}$ for TRITC and TRITC 2 , respectively. The results for TRITC $_{2}$ (unimodal lifetime distribution similar to that of the monomer) indicate that only weak coupling takes place between the two units of the fluorescent dimer molecules, as significant changes in the fluorescence lifetime should be expected for strongly coupled dimers. ${ }^{30}$

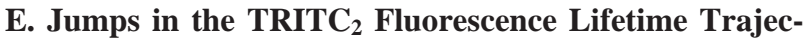
tories. In section $\mathrm{B}$, the influence of orientational effects in the intensity ratio between the two distinct fluorescence intensity levels observed in most of the TRITC $_{2}$ trajectories has been discussed. In that discussion, it was assumed that no quenching of the fluorescence is produced when one of the two dyes in the TRITC $_{2}$ molecule is bleached. In this case, small changes in polarization between the two levels should correlate with intensity ratio values close to 0.5 whereas larger variations imply significantly lower $I_{2} / I_{1}$ values. However, a few of the molecules with small polarization fluctuations $(|\Delta P|<0.1)$ display $I_{2} / I_{1}$ values significantly below 0.5 , namely, $I_{2} / I_{1}<0.4$ for $12 \%\left(\lambda_{\text {exc }}\right.$ $=514 \mathrm{~nm})$ and $26 \%\left(\lambda_{\mathrm{exc}}=550 \mathrm{~nm}\right)$. To determine if this situation arises as a consequence of quenching processes, the change in the fluorescence lifetime for the different intensity levels has been studied. As an example, Figure 9 depicts the fluorescence lifetime trajectory corresponding to the TRITC $_{2}$ molecule whose intensity trajectory has been plotted in Figure $4 \mathrm{~d}$. Clearly, shorter values of the fluorescence lifetime are obtained for the dimmest emissive level: $\langle\tau\rangle=3.2$ and $2.1 \mathrm{~ns}$ 

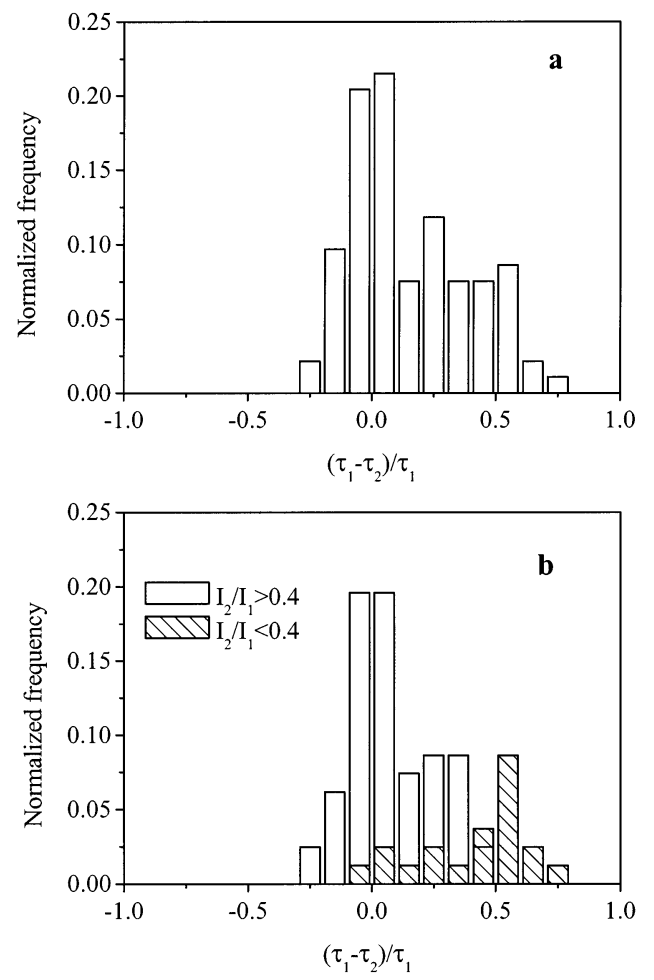

Figure 10. (a) Histogram of the relative differences in fluorescence lifetime $\left(\left(\tau_{1}-\tau_{2}\right) / \tau_{1}\right)$ between the two intensity levels for 93 TRITC $_{2}$ molecules $\left(\lambda_{\text {exc }}=550 \mathrm{~nm}\right.$, power $\left.=4.5 \mathrm{~kW} / \mathrm{cm}^{2}\right)$. (b) Histogram of $\left(\tau_{1}-\tau_{2}\right) / \tau_{1}$ for 68 TRITC $_{2}$ molecules with $|\Delta P|<0.1$ and $I_{2} / I_{1}>0.4$ or $I_{2} / I_{1}<0.4$.

for the first $(0-21 \mathrm{~s})$ and second $(21-51 \mathrm{~s})$ intensity levels, respectively. The $I_{2} / I_{1}$ ratio value for this trajectory is 0.36 .

Figure 10a shows the histogram of relative differences between the lifetimes of both levels $\left(\left(\tau_{1}-\tau_{2}\right) / \tau_{1}\right)$ for 93 TRITC $_{2}$ molecules studied at $\lambda_{\text {exc }}=550 \mathrm{~nm}$. For many molecules, no significant changes occur in the fluorescence lifetime when jumping from the first to the second intensity level. However, there are a considerable number of molecules that deviate from this situation, showing a decrease in $\tau$ as the molecule jumps from the brighter to the dimmer intensity level, as illustrated in Figure 9.

If the decrease in $\tau$ is due to a quenching mechanism, then it must correlate with a deviation of the $I_{2} / I_{1}$ ratio from 0.5 (i.e., those TRITC $_{2}$ molecules for which the first photodamaged dye unit partially quenches the emission of the second one should display a lower $\tau_{2}$ value together with an intensity ratio below 0.5). To prove this, we focused on the subset of $\mathrm{TRITC}_{2}$ molecules not showing orientational effects (68 molecules with $|\Delta P|<0.1)$, and we split their $\left(\tau_{1}-\tau_{2}\right) / \tau_{1}$ histogram into two different components: $I_{2} / I_{1}>0.4(74 \%$, intensity ratio around $0.5)$ and $I_{2} / I_{1}<0.4(26 \%$, intensity ratio clearly below 0.5$)$. The resulting plots are shown in Figure 10b. The molecules with $I_{2} / I_{1}$ values around 0.5 tend to show few changes in the fluorescence lifetime for the two intensity levels (mean value of $\left(\tau_{1}-\tau_{2}\right) / \tau_{1}$ equal to 0.08$)$ whereas significant decreases in $\tau$ correlate with $I_{2} / I_{1}$ ratio values well below 0.5 (on average, $\left(\tau_{1}-\tau_{2}\right) / \tau_{1}$ is equal to 0.42$)$.

The existence of both correlated and uncorrelated behavior between the fluorescence lifetimes and the intensity levels must arise from the existence of at least two distinct mechanisms for photodamaging a chromophore. On one hand, repetitive excitation of the system may yield a photochemically induced derivative of one of the chromophores that is nonabsorbing. In this case, the emission of the second chromophore is not affected: the fluorescence lifetime is unchanged, and in the absence of orientational effects, about half of the initial fluorescence intensity is detected. The nonabsorbing species may appear as a result of photooxidation. Ying and $\mathrm{Xie}^{19}$ called this process photobleaching. On the other hand, the photodegradation of one of the dye units can produce a different species that absorbs the emission of the remaining chromophore, acting as an excitation trap. Then, the emission of the second dye unit is quenched, the fluorescence lifetime decreases, and in absence of orientation effects, less than $50 \%$ of the initial fluorescence is detected. The absorbing-trap formation is believed to be due to the generation of a radical ion by electron transfer. ${ }^{19}$

The existence of these two different photodamaging processes was also observed in a single-molecule study of the allophycocyanin trimer. ${ }^{19}$ However, the correlation between the decrease in the fluorescence lifetime and the intensity ratio for the trapformation situation was not analyzed. In a recent study on single semiconductor nanocrystals, a similar strong correlation between fluorescence intensity fluctuations and fluorescence decay rates has been observed. ${ }^{43}$

\section{Conclusions}

In this work, we have combined ensemble and singlemolecule optical detection to investigate the excitonic behavior of a well-defined multichromophoric system thoroughly: a synthesized dimer of the tetramethylrhodamine-5-isothiocyanate dye (5-TRITC).

Ensemble experiments have shown the existence of two populations of the dimer exhibiting different excitonic interactions: folded conformations give rise to strong coupling between the two dye units, resulting in a nonfluorescent dimer; otherwise, dimer molecules with more extended arrangements are fluorescent, as only weak coupling is established between the chromophores. The equilibrium between weakly and strongly coupled dimer changes with the solvent.

Single-molecule experiments have evidenced some differences in the behavior of monomer and fluorescent dimer molecules. Dimer molecules emit about twice the number of photons emitted by monomers, and they mainly show a twostep photobleaching process instead of the one-step mechanism corresponding to the monomer molecules. The fluorescence lifetime distributions of monomers and dimers are nearly identical, being a clear sign that only weakly coupled TRITC 2 dimers are fluorescent. Furthermore, that fact that the two units in the $\mathrm{TRITC}_{2}$ system are close enough to interact weakly explains the presence of both short and long collective off states, as one of these units being in a dark state (triplet or longer dark state) can efficiently quench the emission from the other one. The special characteristics of the rhodamine/PVA system studied at the single-molecule level (high $Y_{\text {ISC }}$ and large triplet lifetime for rhodamines, low $\mathrm{O}_{2}$ permeability for PVA) allowed us to make a detailed investigation of triplet dynamics in multichromophoric aggregates for the first time. No significant differences were observed between the triplet properties of the monomer and dimer, consistent with the existence of only a weak interaction between the two chromophores in the fluorescent aggregate.

Polarization-sensitive detection in the single-molecule experiments allowed us to distinguish between fluorescent dimer molecules presenting distinct spatial arrangements of the dye units. In agreement with our calculations, weakly coupled dimer molecules with extended (parallel units) and gauche (out-ofplane units) conformations of the linker were experimentally 
detected. Fluorescence lifetime measurements have shown the competition between two different mechanisms of photodamaging the dye units in the dimer: photobleaching to yield a nonabsorbing species and trap formation resulting in partial quenching of the emission coming from the nondamaged dye unit. For the first time, the existence of a correlated lifetime and intensity fluctuations due to trap formation has been shown for a multichromophoric aggregate.

This works demonstrates that accurate control of the spatial arrangement of the chromophores in an aggregate combined with single-molecule detection techniques allows for detailed comprehension of the excitonic behavior in multichromophoric systems.

Acknowledgment. This research has been supported by a Marie Curie Fellowship of the European Community, program IHP-99 under contract number HPMF-CT-2002-01698. J.H. also thanks the Spanish Ministry of Education, Culture and Sports for financial support. We are grateful to Gerald Hebbink for recording the ensemble fluorescence spectra.

\section{References and Notes}

(1) See, for example, van Oijen, A. M.; Ketelaars, M.; Köhler J.; Aartsma, T. J.; Schmidt, J. Science (Washington, D.C.) 1999, 285, 400.

(2) See, for example, Higgins, D. A.; Reid, P. J.; Barbara, P. F. J. Phys. Chem. 1996, 100, 1174.

(3) Bopp, M. A.; Jia, Y. W.; Li, L. Q.; Cogdell, R. J.; Hochstrasser, R. M. Proc. Natl. Acad. Sci. U.S.A. 1997, 94, 10630.

(4) van Oijen, A. M.; Ketelaars, M.; Köhler, J.; Aartsma, T. J.; Schmidt, J. J. Phys. Chem. B 1998, 102, 9363.

(5) Bopp, M. A.; Sytnik, A.; Howard, T. D.; Cogdell, R. J.; Hochstrasser, R. M. Proc. Natl. Acad. Sci. U.S.A. 1999, 96, 11271.

(6) Tietz, C.; Cheklov, O.; Dräbenstedt, A.; Schuster, J.; Wrachtrup, J. J. Phys. Chem. B 1999, 103, 6328.

(7) Tietz, C.; Jelezko, F.; Gerken, U.; Schuler, S.; Schubert, A.; Rogl, H.; Wrachtrup, J. Biophys. J. 2001, 81, 556.

(8) Wu, M.; Goodwin, P. M.; Ambrose, W. P.; Keller, R. A. J. Phys. Chem. 1996, 100, 17406.

(9) Hofkens, J.; Schroeyers, W.; Loos, D.; Cotlet, M.; Köhn, F.; Vosch, T.; Maus, M.; Herrmann, A.; Müllen, K.; Gensch, T.; De Schryver, F. C. Spectrochim. Acta, Part A 2001, 57, 2093.

(10) Jelezko, F.; Tietz, C.; Gerken, U.; Wrachtrup, J.; Bittl, R. J. Phys. Chem. B 2000, 104, 8093.

(11) Vanden Bout, D. A.; Yip, W.-T.; Hu, D.; Fu, D.-K.; Swager, T. M.; Barbara, P. F. Science (Washington, D.C.) 1997, 277, 1071.

(12) Yip, W.-T.; Hu, D.; Yu, J.; Vanden Bout, D. A.; Barbara, P. F. J. Phys. Chem. A 1998, 102, 7564.

(13) Hu, D.; Yu, J.; Barbara, P. F. J. Am. Chem. Soc. 1999, 121, 6936.

(14) Yu, J.; Hu, D. H.; Barbara, P. F. Science (Washington, D.C.) 2000, $289,1327$.

(15) Huser, T.; Yan, M.; Rothberg, L. J. Proc. Natl. Acad. Sci. U.S.A. 2000, 97, 11187.

(16) Cotlet, M.; Hofkens, J.; Köhn, F.; Habuchi, S.; Dirix, G.; van Guyse, M.; Michiels, J.; Vanderleyden, J.; De Schryver, F. C. Chem. Phys. Lett. 2001, 336, 415.

(17) Lounis, B.; Deich, J.; Rosell, F. I.; Boxer, S. G.; Moerner, W. E. J. Phys. Chem. B 2001, 105, 5048 .
(18) García-Parajó, M. F.; Koopman, M.; van Dijk, E. M. H. P.; Subramaniam, V.; van Hulst, N. F. Proc. Natl. Acad. Sci. U.S.A. 2001, 98 14392.

(19) Ying, L.; Xie, X. S. J. Phys. Chem. B 1998, 102, 10399.

(20) Deniz, A. A.; Dahan, M.; Grunwell, J. R.; Ha, T.; Faulhaber, A. E.; Chemla, D. S.; Weiss, S.; Schultz, P. G. Proc. Nat. Acad. Sci. U.S.A. 1999, 96, 3670 .

(21) Hofkens, J.; Maus, M.; Gensch, T.; Vosch, T.; Cotlet, M.; Köhn, F.; Herrmann, A.; Müllen, K.; De Schryver, F. C. J. Am. Chem. Soc. 2000, $122,9278$.

(22) Gensch, T.; Hofkens, J.; Köhn, F.; Vosch, T.; Heirmann, A.; Müllen, K.; De Schryver, F. C. Single Mol. 2001, 1, 35.

(23) Vosch, T.; Hofkens, J.; Cotlet, M.; Köhn, F.; Fujiwara, H.; Groenheid, R.; Van Der Biest, K.; Weil, T.; Herrmann, A.; Müllen, K.; Mukamel, S.; Van der Auweraer, M.; De Schryver, F. C. Angew. Chem., Int. Ed. 2001, 40, 4643.

(24) Gronheid, R.; Hofkens, J.; Köhn, F.; Weil, T.; Reuther, E.; Müllen, K.; De Schryver, F. C. J. Am. Chem. Soc. 2001, 124, 2418.

(25) Christ, T.; Petzke, F.; Bordat, P.; Herrmann, A.; Reuther, E.; Müllen, K.; Basché, T. J. Lumin. 2002, 98, 23.

(26) Frisch, M. J.; Trucks, G. W.; Schlegel, H. B.; Scuseria, G. E.; Robb, M. A.; Cheeseman, J. R.; Zakrzewski, V. G.; Montgomery, J. A., Jr.; Stratmann, R. E.; Burant, J. C.; Dapprich, S.; Millam, J. M.; Daniels, A. D.; Kudin, K. N.; Strain, M. C.; Farkas, O.; Tomasi, J.; Barone, V.; Cossi, M.; Cammi, R.; Mennucci, B.; Pomelli, C.; Adamo, C.; Clifford, S.; Ochterski, J.; Petersson, G. A.; Ayala, P. Y.; Cui, Q.; Morokuma, K.; Malick, D. K.; Rabuck, A. D.; Raghavachari, K.; Foresman, J. B.; Cioslowski, J.; Ortiz, J. V.; Stefanov, B. B.; Liu, G.; Liashenko, A.; Piskorz, P.; Komaromi, I.; Gomperts, R.; Martin, R. L.; Fox, D. J.; Keith, T.; Al-Laham, M. A.; Peng, C. Y.; Nanayakkara, A.; Gonzalez, C.; Challacombe, M.; Gill, P. M. W.; Johnson, B. G.; Chen, W.; Wong, M. W.; Andres, J. L.; Head-Gordon, M.; Replogle, E. S.; Pople, J. A. Gaussian 98, revision A.7; Gaussian, Inc.: Pittsburgh, PA, 1998.

(27) Vallée, R.; Tomczack, N.; Gersen, H.; van Dijk, E. M. H. P.; GarcíaParajó, M. F.; Vancso, G. J.; van Hulst, N. F. Chem. Phys. Lett. 2001, 348, 161.

(28) Kemnitz, K.; Yoshihara, K. J. Phys. Chem. 1991, 95, 6095.

(29) del Monte, F.; Levy, D. J. Phys. Chem. B 1999, 103, 8080.

(30) Kasha, M.; Rawls, H. R.; Ashaf El-Bayoumi, M. Pure Appl. Chem. 1965, 11,371 .

(31) Davydov, A. S. Theory of Molecular Exciton; McGraw-Hill: New York, 1962.

(32) Förster, T. In Modern Quantum Chemistry; Sinanoglu, O., Ed.; Academic Press: New York, 1965.

(33) Dexter, D. L. J. Chem. Phys. 1953, 21, 836.

(34) Hsu, C.-P.; Walla, P. J.; Head-Gordon, M.; Fleming, G. R. J. Phys. Chem. 2001, 105, 11016.

(35) Liang, K.; Farahat, M. S.; Perlstein, J.; Law, K.-Y.; Whitten, D. G. J. Am. Chem Soc. 1997, 119, 830.

(36) Lu, L.; Lachicotte, R. J.; Penner, T. L.; Perlstein, J.; Whitten, D. G. J. Am. Chem Soc. 1999, 121, 8146.

(37) Moerner, W. E.; Orrit, M. Science (Washington, D.C.) 1999, 283, 1670 .

(38) Weiss, S. Science (Washington, D.C.) 1999, 283, 1676

(39) Lu, H. P.; Xie, X. S. Nature (London) 1997, 385, 143.

(40) Ha, T.; Enderle, T.; Chemla, D. S.; Selvin, P. R.; Weiss, S. Chem. Phys. Lett. 1997, 271, 1.

(41) Köhn, F.; Hofkens, J.; De Schryver, F. C. Chem. Phys. Lett. 2000, 321,373 .

(42) Veerman, J. A.; García-Parajó, M. F.; Kuipers, L.; van Hulst, N. F. Phys. Rev. Lett. 1999, 83, 2155.

(43) Schlegel, G.; Bohnenberger, J.; Potapova, I.; Mews, A. Phys. Rev. Lett. 2002, 88, 137401. 